\title{
Factors associated with awareness and utilisation of a community mobilisation intervention for female sex workers in Andhra Pradesh, India
}

\author{
Kim M Blankenship, Rebekah Burroway, Elizabeth Reed
}

\begin{abstract}
- Supplementary appendix is published online only at http:// sti.bmj.com/content/vol86/ issue 1
\end{abstract}

Duke University, Durham, North Carolina, USA

\section{Correspondence to} Kim M Blankenship, Department of Sociology and Duke Global Health Institute, Duke University, PO Box 90519, Durham, NC 27708, USA:

kim.blankenship@duke.edu

Accepted 30 October 2009

\section{UNLOCKID}

This paper is freely available online under the BMJ Journals unlocked scheme, see http://sti. bmi.com/site/about/unlocked. xhtml.

\section{ABSTRACT}

Objectives Examine factors associated with awareness and active utilisation of a community mobilisation intervention (CMI) to address HIV risk in female sex workers (FSWs) in a context characterised by multiple forms of sex work.

Design Data came from two rounds, conducted in Spring 2006 and Spring 2007, of a serial cross-sectional survey of FSWs ( $n=812$ in round $1, n=673$ in round 2) recruited through respondent-driven sampling in Rajahmundry, Andhra Pradesh, India.

Methods Descriptive statistics compared characteristics of programme aware and unaware FSWs and from among the aware, to characterise active program users. Multinomial logistic regression was used to assess factors associated with programme exposure.

Results Between Rounds 1 and 2, programme awareness increased from $41.8 \%$ to $69.6 \%$ of respondents, and active utilisation lamong those who were aware) increased from $49.2 \%$ to $61.0 \%$. Streetbased FSWs were under-represented and brothel-based FSWs overrepresented in both groups and rounds. Geographic proximity and literacy were associated with programme awareness but not utilisation. The most important factor associated with both forms of intervention exposure across rounds was willingness to be identified in public as a FSWs (OR 2.2-4.8).

Conclusion Public visibility is a critical component of CMls. Such interventions should develop strategies for involving FSWs that allow them to remain invisible, while also working to reduce the threat associated with public visibility. In contexts where sex work occurs in multiple venues, it is important to develop CMls that include and address the needs of FSWs working in them all.

\section{INTRODUCTION}

Female sex workers (FSWs) comprise one of several groups at particular risk for HIV in India and globally. For example, in southern India, where HIV rates are among the country's highest, prevalence among FSWs is estimated to be $14.5 \%$, ranging across districts from $2-38 \%{ }^{1}$ Much has been written about the impact of different interventions for addressing HIV risk in FSWs. ${ }^{2}$ In India, such interventions have been shown to be associated with FSW's knowledge of HIV risks, ${ }^{3}$ consistent condom use with clients, ${ }^{4} 5$ condom use with occasional clients, repeat clients and regular partners, ${ }^{6}$ and sexually transmitted infection (STI) prevalence. ${ }^{6}$ If target coverage is achieved, they have the potential to prevent a substantial number of new infections in FSWs and the general population. ${ }^{7}$
Given not only their ability to address HIV among FSWs, but their potential to affect the dynamics of HIV prevention across the country, it is important to consider the extent to which such interventions are reaching FSWs and whether some groups of FSWs are more likely than others to have access to their benefits. Steen et $a l^{8}$ have shown that as a result of STI interventions implemented as part of the Bill and Melinda Gates Foundation's Avahan India AIDS Initiative (hereafter referred to as Avahan), nearly three-quarters (70\%) of FSWs in the Avahan coverage area (primarily southern India) have been contacted through peer outreach and $41 \%$ have visited an STI clinic at least once. However, whether all groups of FSWs are represented in these number is not clear from their study.

The extent and representativeness of coverage is particularly important for community mobilisation interventions (CMIs), a form of structural interventions that expressly aims to organise FSWs for collective action, to challenge the power inequalities that promote their HIV risk. ${ }^{9}{ }^{10}$ In theory, the full power of CMIs is achieved when all FSWs are organised to address their shared interests. This may be particularly challenging in a context such as southern India where sex work takes many different forms. ${ }^{11}$ While all sex workers likely share some common experiences as sex workers, it is also likely that their interests, needs and vulnerabilities vary depending on, among other things, the venues in which they work. For example, compared to other groups of FSWs, those who work from their homes may face less police harassment ${ }^{10}$ but also have less access to a range of health services, including HIV prevention. Similarly, different categories of FSWs may be more or less available to intervention outreach workers and peer educators. Yet arguably, given their overall objective of collective organisation and representation, and regardless of their impact on HIV-related risk, one measure of the success of CMIs is whether they adequately include and represent all FSWs.

Research has documented a variety of challenges associated with implementation of CMIs. ${ }^{12-14}$ However, this work has not systematically examined whether these interventions have reached all groups of FSWs or whether all groups are represented among intervention participants (eg, as peer educators or advocates). Yet knowing this is important for assessing the potential overall impact of these interventions on the pandemic. In addition, CMIs may create new dimensions of marginalisation among FSWs (eg, those with access to intervention benefits and those without) if certain 
FSWs are systematically left out of them. This in turn not only undermines the goal of collectivisation but also, more specifically, can hinder HIV prevention (eg, more marginalised groups of FSWs may be willing to accept more money for condomless sex making it hard to enforce norms of $100 \%$ condom use). Also, CMIs that do not adequately represent the interests of all FSWs may not be sustainable.

In this paper, we address these issues by analysing exposure to a CMI implemented among FSWs in the Rajahmundry area of Andhra Pradesh. The CMI, which is part of Avahan and is implemented by the Indian branch of an international nongovernmental organisation, focuses simultaneously on organising FSWs to build collective power and directing this collective power towards addressing structural factors that produce HIV risk among FSWs, such as stigma, policing policy and practice, condom availability, availability of STI services and FSW economic vulnerability. Social change agents are identified from among local FSWs to serve as both peer educators and community organisers. As such, they engage in traditional intervention activities, such as health education and condom distribution, as well as community organising activities, such as encouraging FSWs to join together as other worker groups have done to enhance their power. As part of the intervention, mobilised FSWs engage in a variety of activities, including leading or attending public rallies, meeting with the media, public officials and local community groups to raise awareness of the issues faced by FSWs, advising and even participating in the operations of intervention-run STI clinics, and responding to incidents of police brutality against FSWs. The CMI also encourages the organising of community based organisations comprised of and led by FSWs. Most of these activities require a high degree of visibility for intervention-involved FSWs.

In this paper, we analyse the extent of and factors associated with exposure to the CMI among FSWs in the intervention's operating area. Specifically, we define two dimensions of intervention exposure: awareness of the intervention and active utilisation of or participation in its services and activities. We hypothesise that it is likely that two different types of factors will be associated with these different dimensions of exposure. First, whether FSWs are exposed to the intervention should be a function of programme activities to reach them. FSWs may learn of the intervention because they have seen materials produced by it or because they have read or heard about it in the media. Direct, face-to-face exposure requires that the intervention engages in activities through which they come in contact with FSWs. Thus, it is likely that intervention exposure among FSWs will reflect such factors as geography (primary intervention coverage area), venues in which FSWs work (with some venues such as brothels likely to be easier for intervention workers to identify and locate FSWs) and, perhaps literacy, particularly for those who have not had face-to-face contact.

Programme exposure that goes beyond simply hearing about the intervention to using its services or participating in its activities likely reflects not only programme efforts but, also, the willingness and ability of FSWs to get involved. This, in turn, may reflect their socioeconomic and work vulnerabilities. For example, given the high degree of visibility required for full participation in the CMI, it is likely that FSWs fearful of such exposure will be less inclined to become involved. Various dimensions of FSW vulnerability may also impact their likelihood of becoming involved in a CMI. From the standpoint of impact on the HIV pandemic, it is important that FSWs engaging in the riskiest behaviours are exposed to and engaged with the intervention. However, it is often the case that FSWs at greatest risk for HIV are also particularly vulnerable-for example, lacking economic security and autonomy over their work. ${ }^{9}$ They may have both the most to gain (to the extent that the CMI addresses these aspects of their vulnerability) and the most to lose (to the extent that involvement with the intervention takes them away from their income generating work or leads to repercussions from police, clients, partners or other powerbrokers) from involvement with CMIs.

Factors associated with exposure to a CMI also may vary over time. Most generally, if the intervention is successful, as it matures, a higher proportion of FSWs in its operating area will be both aware of and actively involved with it. Further, even if FSWs working in some venues are more likely than others to be contacted early in the intervention's history, as the network of intervention-exposed FSWs increases, it is reasonable to expect that venue will not be associated with intervention awareness over time.

Clearly, there are a variety of factors that may be associated with awareness and utilisation of a CMI, including such things as geographic proximity, literacy, willingness to be open about being an FSW and degree of vulnerability, and these may vary over time. Understanding their relationship to intervention exposure has important implications for intervention representativeness, sustainability and success, and for overall epidemic impact as well. Here, we analyse the extent of and factors associated with two types of exposure to a CMI and then discuss the implications of our findings for CMIs aimed at reducing HIV risk among FSWs in settings with multiple types of sex work

\section{METHODS}

\section{Data}

Data for this cross-sectional analysis were collected as part of Project Parivartan-a larger study of the implementation of a CMI and its impact on vulnerability to HIV risk among FSWs in the Rajahmundry area of the East Godavari district of Andhra Pradesh. The CMI is being implemented by an Indian non-governmental organisation with international headquarters in the United States. We analysed survey data collected in two rounds: the first conducted from April to June $2006(n=812)$, about a year after the intervention had been implemented, and the second conducted from March to May 2007 ( $n=673$ ). Eligible participants were female, at least $18 \mathrm{y}$ of age and reported having exchanged sex for money at least once in the year prior to the survey. For both data collection rounds, participants were recruited via respondent-driven sampling-a modified chain referral technique designed to recruit hidden populations. ${ }^{15} 16$ Initially, five seeds representing different groups of FSWs completed the survey. They were then asked to distribute up to three coupons to other FSWs in their social networks who met the study's eligibility criteria. Subsequent participants who came into the study with a coupon were similarly given the opportunity to distribute coupons to up to three members of their networks. The survey was anonymous, so we were not able to distinguish those who participated in both waves of data collection. (See Web Appendix for a more extensive discussion.) Given the high number of waves (ie, each seed recruited women who recruited more women and this continued for multiple waves), we believe that each round represented an adequate coverage of the population of FSWs in this region. ${ }^{17}$ Further, the fact that a substantial number of women who participated in the survey had not heard of the intervention (see below) suggests that our sampling strategy provided access to a broader group of FSWs in the area than the intervention did. 
Interviews of 90-120 min in length were conducted in the local language (Telugu) by trained interviewers who ensured participants' informed consent. Participants received modest compensation for completion of the survey as well as for successfully recruiting other FSWs into the study. Participants missing data on relevant study variables were excluded from all analyses, resulting in $n=788$ in round 1 and $n=622$ in round 2 . The research protocol was approved by the Duke University Health Systems Institutional Review Board, Yale University's Human Investigations Committee and the VHS-YRG Care Medical Centre Institutional Review Board in Chennai.

\section{Variables \\ Programme exposure}

We considered two distinct dimensions of exposure to the CMI. We measured awareness of the intervention by asking respondents if they had ever heard of the intervention. Respondents were coded as active intervention users if, in the last 6 months, they had done any of the following: gone to or tried to bring other sex workers to an intervention sponsored drop-in centre, received STI treatment or sought general healthcare at an intervention clinic, or went to the police to speak for the rights of sex workers.

\section{Sample characteristics}

Age was measured continuously. We developed a dichotomous variable to indicate having any children. Marital status was measured as married, versus never married or separated/ divorced/widowed. Geographic proximity to the intervention was indicated by whether a respondent lived in the primary intervention coverage area (yes/no). Literacy was measured as the ability to read or write (yes/no).

\section{Economic vulnerability}

We assessed economic vulnerability with a continuous living environment variable based on whether a respondent indicated that she currently lived in a home with electricity, running water, bathrooms/toilet or telephones (values range from 0-4); and through a measure of debt based on whether the respondent reported current debt (yes/no).

\section{Control over work}

We assessed two aspects of control over work: deciding the type of sex with a client and deciding the amount of money to charge the client. Control was indicated by deciding sometimes, usually or always, as compared to never or rarely.

\section{Other work-related vulnerabilities}

We included brothel, agriculture and street to indicate venues in which respondents worked (eg, whether work in a brothel (yes/ no)). We measured the frequency of work through a dichotomous variable that indicates a high frequency of sex trades based on whether respondents reported a number of sex trades greater than the sample median in the last 7 days (median $=8$ in round 1 ; median $=9$ in round 2). Police vulnerability was indicated by whether or not a respondent had ever been arrested (yes/no). Work-related violence was assessed by asking respondents if clients or police had beaten them (hit, slapped, pushed, kicked, punched, choked or burned), threatened or used a weapon against them, or forced them to have sex against their will (oral, vaginal or anal) in the last 6 months. We also measured whether a respondent was willing to be identified in public by combining responses to two distinct questions. Respondents were coded as willing to be identified in public if they responded affirmatively to either of the following questions, asked one after the other: "In the last 6 months, have you attended any public events (such as a rally or gathering of sex workers) where you could have been identified as a sex worker?" And if she said "no," she was asked, "Are you willing to attend a public event where you could be identified as a sex worker?"

\section{Estimation technique}

We used descriptive statistics to compare the characteristics of programme aware and unaware FSWs, and among programme aware, to characterise active intervention utilisers. We used multinomial logistic regression to assess factors associated with (1) awareness of and (2) active participation in the intervention. First, we identified possible factors associated with intervention exposure by conducting a series of bivariate analyses; relevant sample characteristics that had an association at the $p<0.10$ level with either programme exposure variable in round 1 or round 2 were included in the adjusted analyses. To examine active participation in the intervention, in contrast to intervention awareness, we conducted the analyses among a subset of the sample who reported being aware of the intervention $(n=329$ for round $1, n=433$ for round 2 ). Final models are adjusted for sample characteristics and all other independent variables. We present findings for both rounds of data collection separately. In addition, we discuss differences in significant findings between rounds. All analyses were conducted using STATA/SE version 9.

\section{RESULTS}

In round 1 of the survey, $41.8 \%$ of respondents indicated that they were aware of the intervention; $1 \mathrm{y}$ later, $69.6 \%$ of respondents reported being aware of it (table 1). Active utilisation increased similarly, with $49.2 \%$ of those who had heard of the intervention reporting active utilisation in round 1 and $61.0 \%$ in round 2 (table 2 ).

As table 1 indicates, there are a number of characteristics that distinguish FSWs who were aware of the intervention from those who were not (age (round 2), marital status (round 1), geographic proximity, literacy and venue). Among those who were aware of the intervention, there were fewer differences in sample characteristics between those who reported active utilisation and those who did not (table 2).

Findings from the final logistic regression models for round 1 indicate that, compared to those with no intervention exposure, those who reported awareness of the intervention were more likely to report the following: a high frequency of sex trades (OR $1.5,95 \%$ CI 1.1 to 2.1), a better living environment (OR 1.4, 95\% CI 1.2 to 1.6), willingness to be publicly identified as a sex worker (OR 2.2, 95\% CI 1.6 to 3.2), having ever been arrested (OR 2.0, 95\% CI 1.4 to 3.0) and control over the amount of money made with clients (OR 1.8, 95\% CI 1.3 to 2.6) (table 3). Findings were similar for round 2 , except that slightly fewer of these variables were significant and willingness to be identified in public was even more strongly associated with intervention awareness (OR 4.8, 95\% CI 3.1 to 7.3) (table 3).

Among those who reported awareness of the intervention, the final logistic regression models indicate that those actively using the intervention in round 1 were more likely to report willingness to be identified in public (OR $3.4,95 \%$ CI 1.9 to 6.2 ), ever being arrested (OR 2.0, 95\% CI 1.2 to 3.4) and control over the amount of money made with clients (OR 1.8, 95\% CI 1.1 to 3.1) (table 4). Findings from round 2 were similar, except high frequency of sex trades (OR 1.6, 95\% CI 1.0 to 2.5) was also significantly associated with active involvement and willingness 
Table 1 Sample characteristics, ${ }^{*}$ by awareness of the intervention

\begin{tabular}{|c|c|c|c|c|c|c|c|c|}
\hline & \multicolumn{4}{|c|}{ Round $1(n=788)$} & \multicolumn{4}{|c|}{ Round $2(n=622)$} \\
\hline & \multicolumn{2}{|c|}{$\begin{array}{l}\text { CMI awareness } \\
(n=329 ; 41.8 \%)\end{array}$} & \multicolumn{2}{|c|}{$\begin{array}{l}\text { No exposure } \\
(\mathrm{n}=459 ; 58.2 \%)\end{array}$} & \multicolumn{2}{|c|}{$\begin{array}{l}\text { CMI awareness } \\
(n=433 ; 69.6 \%)\end{array}$} & \multicolumn{2}{|c|}{$\begin{array}{l}\text { No exposure } \\
(\mathrm{n}=189 ; 30.4 \%)\end{array}$} \\
\hline & $\mathbf{N}$ & $\%$ & $\mathbf{N}$ & $\%$ & $\mathbf{N}$ & $\%$ & $\mathbf{N}$ & $\%$ \\
\hline \multicolumn{9}{|l|}{ Age, y } \\
\hline Less than 25 & 50 & 15.2 & 87 & 19.0 & 50 & 11.6 & 31 & 16.4 \\
\hline $25-29$ & 70 & 21.3 & 91 & 20.0 & 85 & 19.6 & 44 & 23.3 \\
\hline $30-34$ & 59 & 17.9 & 94 & 20.5 & 92 & 21.3 & 41 & 21.7 \\
\hline $35-39$ & 86 & 26.1 & 99 & 21.6 & 99 & 22.9 & 38 & 20.1 \\
\hline $40-44$ & 32 & 9.7 & 43 & 9.4 & 52 & 12.0 & 19 & 10.1 \\
\hline $45-49$ & 22 & 6.7 & 22 & 4.8 & 36 & 8.3 & 9 & 4.8 \\
\hline $50+$ & 10 & 3.0 & 23 & 5.0 & 19 & 4.4 & 7 & 3.7 \\
\hline & \multicolumn{4}{|c|}{$p=0.437$} & \multicolumn{4}{|c|}{$\mathrm{p}=0.017$} \\
\hline Children & 287 & 87.2 & 419 & 91.3 & 386 & 89.2 & 177 & 93.7 \\
\hline & \multicolumn{4}{|c|}{$p=0.066$} & \multicolumn{4}{|c|}{$p=0.078$} \\
\hline Currently married & 51 & 15.5 & 118 & 25.7 & 99 & 22.9 & 31 & 16.4 \\
\hline & \multicolumn{4}{|c|}{$p=0.0001$} & \multicolumn{4}{|c|}{$p=0.069$} \\
\hline Geographic proximity & 277 & 84.2 & 361 & 78.7 & 373 & 86.1 & 146 & 77.3 \\
\hline & \multicolumn{4}{|c|}{$\mathrm{p}=0.051$} & \multicolumn{4}{|c|}{$p=0.006$} \\
\hline Literacy & 101 & 30.7 & 74 & 16.1 & 150 & 34.6 & 29 & 15.3 \\
\hline & \multicolumn{4}{|c|}{$p=0.000$} & \multicolumn{4}{|c|}{$p=0.000$} \\
\hline \multicolumn{9}{|l|}{ Venue } \\
\hline Agriculture & 23 & 7.0 & 68 & 14.8 & 23 & 5.3 & 10 & 5.3 \\
\hline & \multicolumn{4}{|c|}{$p=0.001$} & \multicolumn{4}{|c|}{$\mathrm{p}=0.992$} \\
\hline Brothel & 26 & 7.9 & 15 & 3.3 & 65 & 15.0 & 16 & 8.5 \\
\hline & \multicolumn{4}{|c|}{$p=0.004$} & \multicolumn{4}{|c|}{$p=0.026$} \\
\hline Street & 28 & 8.5 & 83 & 18.1 & 56 & 12.9 & 54 & 28.6 \\
\hline & \multicolumn{4}{|c|}{$\mathrm{p}=0.000$} & \multicolumn{4}{|c|}{$p=0.000$} \\
\hline
\end{tabular}

*Numbers refer to those who responded 'yes'.

$\mathrm{CMI}$, community mobilisation intervention.

to be identified in public had an even stronger association (OR $3.6,95 \%$ CI 2.0 to 6.5$)$.

\section{DISCUSSION}

Our findings indicate that in many ways, the CMI has been successful in gaining exposure to a relatively large and diverse group of FSWs, especially as it has matured. A little more than $2 \mathrm{y}$ after it started, more than two-thirds of survey respondents indicated that they were aware of it and almost two-thirds of these FSWs indicated that they were actively involved in the intervention. To the extent that awareness of and, especially, active involvement in a CMI reduces HIV-related risk among FSWs, ${ }^{4}$ this has important implications for HIV prevention among this population. At the same time, factors associated with these two different forms of exposure suggest how the CMI has achieved this success as well as where it has fallen short and what may be necessary for increasing intervention exposure among FSWs. Insofar as these findings are generaliseable, they have implications for CMIs for HIV prevention that are being implemented in other settings where sex work takes diverse forms.

CMIs, such as those implemented as part of Avahan for HIV prevention in FSWs, reflect the view that risk for disease is a product of inequalities of power that, among other things, constrain FSWs' ability to engage in protective behaviours. Collective mobilisation represents a means of challenging these power inequalities, but it requires that FSWs organise together as sex workers and demand changes in the context that promotes their risk. As such, CMIs involve a high degree of visibility-collectively mobilised FSWs confront or negotiate with police and other officials, organise public rallies, other media events, and their own organisations, and so on. Reflecting this, our data show that, more than any other factor, willingness to be exposed publicly is critical in both reaching and actively involving FSWs in the CMI, and appears to become more important over time.

Beyond public visibility, our data show that factors with implications for intervention activities to reach FSWs are associated with programme awareness. Not surprisingly, FSWs who are aware of the intervention are more likely than unaware FSWs to live in geographic proximity to it. Street-based FSWs are consistently under-represented and brothel-based FSWs over represented among programme aware, perhaps also indicating the degree to which these different groups are identifiable to intervention workers. Finally, literacy is associated with programme awareness, perhaps reflecting the fact that FSWs who do not hear of the intervention through face-to-face contact must be able to read about it in printed materials or media reports.

Vulnerability is associated with intervention awareness in different ways, depending on the type of vulnerability. Consistent with the finding regarding literacy, in terms of socioeconomic status and control over work, it is less vulnerable FSWs who are most likely to be aware of the intervention. However, FSWs who are more vulnerable sexually (higher frequency of sex trade), and in relation to police arrest, are more likely to be aware of the intervention. These findings change over time, with sexual vulnerability and control over work not significant by round 2. It may be that the intervention focused its efforts first on identifying those FSWs deemed at greatest risk for HIV (high frequency of sex exchanges), but over time has expanded their efforts to other FSWs. It is also the case that the intervention has devoted considerable effort to addressing policing practices, including ensuring that proper arrest procedures are followed, ${ }^{12}$ which may raise awareness among FSWs who are more vulnerable to arrest. 
Table 2 Sample characteristics, ${ }^{*}$ by active utilisation of the intervention

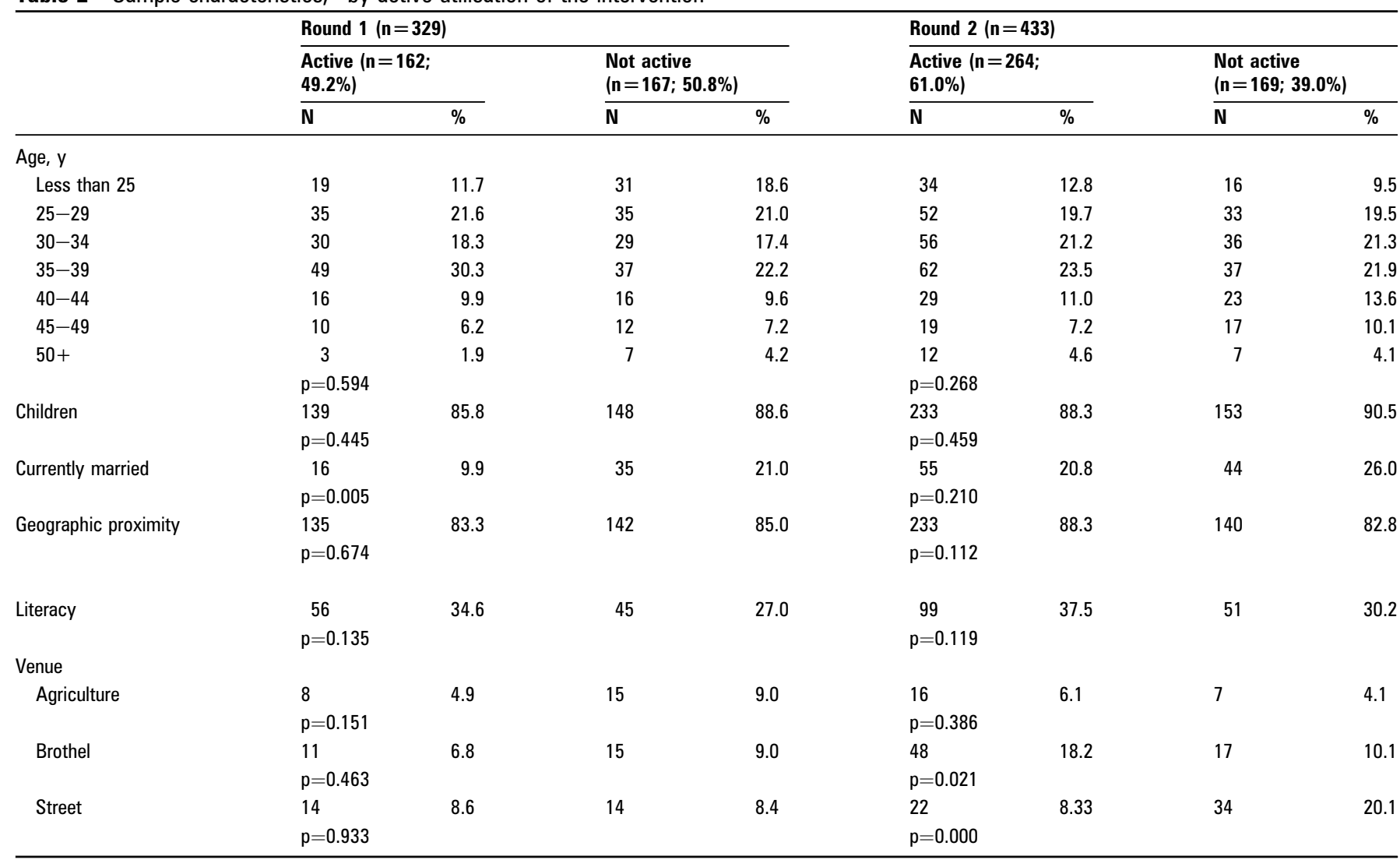

*Numbers refer to those who responded 'yes'.

Measures associated with intervention reach (geographic proximity, literacy) are not associated with active involvement in the intervention. Beyond willingness to be publicly identified as a FSW, venue (brothel and street), high frequency of sex trades and being arrested are associated with active involvement. We suspect, but cannot confirm with these data, that some of these differences between rounds may reflect a process in which intervention activities are directed towards the issues of greatest concern to those who are participating in it. This, in turn, causes FSWs for whom these are also major issues and who, therefore, perceive themselves to benefit from active participation to become more involved. For example, perhaps one indication of the success of the intervention's emphasis on policing is the degree to which FSWs who have been arrested become involved in it. At the same time, this may reduce the likelihood that FSWs who do not face police problems to the same degree will actively participate. A further consequence of the police (and media) advocacy activity of the intervention is that it, along with the FSWs who are actively involved with it, becomes increasingly visible. FSWs who are not willing to be publicly visible may become even less inclined to actively participate; thereby, enhancing the association between active participation and willingness to be identified in public as a sex worker.

What are the implications of this analysis for CMIs more generally? CMIs require public visibility of FSWs. To ensure that they involve and represent the needs of FSWs who fear public visibility, CMIs need to create opportunities for these FSWs to participate without being visible. They need to direct efforts to

Table 3 OR of the likelihood of intervention awareness

\begin{tabular}{|c|c|c|c|c|c|c|}
\hline & \multicolumn{3}{|c|}{ Round 1 ( $n=788$ ) } & \multicolumn{3}{|c|}{ Round 2 ( $n=622$ ) } \\
\hline & $\%$ & Adjusted + OR (CI) & Final model $\neq$ OR (CI) & $\%$ & Adjusted $†$ OR (Cl) & Final model $\neq$ OR $(\mathrm{Cl})$ \\
\hline High frequency of sex trades & 48.6 & $1.8^{* * *}(1.3$ to 2.4$)$ & $1.5^{*}(1.1$ to 2.1$)$ & 49.0 & $1.3(0.9$ to 1.8$)$ & $1.0(0.6$ to 1.5$)$ \\
\hline \multicolumn{7}{|l|}{ Economic vulnerability } \\
\hline Living environment & & $1.4^{* * *}(1.2$ to 1.6$)$ & $1.4^{* * *}(1.2$ to 1.6$)$ & & $1.4^{* * *}(1.2$ to 1.6$)$ & $1.4^{* * *}(1.2$ to 1.7$)$ \\
\hline Debt & 83.6 & $1.7^{*}(1.1$ to 2.7$)$ & $1.6(1.0$ to 2.6$)$ & 80.4 & $1.6^{*}(1.0$ to 2.4$)$ & $1.4(0.9$ to 2.3$)$ \\
\hline Willing to be identified in public & 60.9 & $2.8^{* * *}(2.0$ to 3.8$)$ & $2.2^{* * *}(1.6$ to 3.2$)$ & 72.8 & $5.1^{* * *}(3.4$ to 7.6$)$ & $4.8^{* * *}(3.1$ to 7.3$)$ \\
\hline Arrested & 26.5 & $3.1^{* * *}(2.2$ to 4.4$)$ & $2.0^{* * *}(1.4$ to 3.0$)$ & 29.1 & $2.7^{* * *}(1.7$ to 4.3$)$ & $1.8^{*}(1.1$ to 3.0$)$ \\
\hline Work-related violence & 17.9 & $1.9 * *(1.3$ to 2.8$)$ & 1.2 (0.8 to 1.8$)$ & 14.5 & $1.9 *(1.1$ to 3.4$)$ & $1.6(0.9$ to 3.1$)$ \\
\hline \multicolumn{7}{|l|}{ Control over work } \\
\hline Type of sex & 53.6 & $1.6^{* *}(1.2$ to 2.2$)$ & $1.3(1.0$ to 1.9$)$ & 75.2 & $1.1(0.7$ to 1.7$)$ & $1.0(0.6$ to 1.7$)$ \\
\hline Amount of money & 53.7 & $2.3^{* * *}(1.7$ to 3.1$)$ & $1.8^{* * *}(1.3$ to 2.6$)$ & 70.7 & $1.5^{*}(1.0$ to 2.3$)$ & $1.2(0.8$ to 1.9$)$ \\
\hline
\end{tabular}

${ }^{*} \mathrm{p}<0.05{ }^{* * *} \mathrm{p}<0.01{ }^{* * *} \mathrm{p}<0.001$.

†Adjusted $\mathrm{OR}$ control for sample characteristics (age, lack of children, married, geographic proximity, literacy and venue).

†Final models include sample characteristics as well as all other variables listed here. 
Table 4 OR of the likelihood of actively using the intervention

\begin{tabular}{|c|c|c|c|c|c|c|}
\hline & \multicolumn{3}{|c|}{ Round $1(n=329)$} & \multicolumn{3}{|c|}{ Round $2(n=433)$} \\
\hline & $\overline{\%}$ & Adjusted $†$ OR (CI) & Final model $\neq$ OR (Cl) & $\overline{\%}$ & Adjusted $†$ OR (CI) & Final model $\neq$ OR (CI) \\
\hline High frequency of sex trades & 57.1 & $1.4(0.9$ to 2.1$)$ & $1.2(01.7$ to 1.9$)$ & 50.4 & $2.1^{* * *}$ (1.4 to 3.2$)$ & $1.6^{*}(1.0$ to 2.5$)$ \\
\hline Living environment & & $1.1(0.9$ to 1.4$)$ & $1.1(0.9$ to 1.4$)$ & & $1.0(0.9$ to 1.2$)$ & $1.1(0.9$ to 1.3$)$ \\
\hline Debt & 89.1 & $1.0(0.5$ to 2.1$)$ & $0.9(0.4$ to 2.0$)$ & 83.8 & $1.1(0.7$ to 1.9$)$ & 0.9 (0.5 to 1.6$)$ \\
\hline Willing to be identified in public & 73.25 & $4.2^{* * *}$ (2.4 to 7.4$)$ & $3.4^{* * *}(1.9$ to 6.2$)$ & 83.1 & $4.3^{* * *}(2.4$ to 7.5$)$ & $3.6^{* * *}(2.0$ to 6.5$)$ \\
\hline \multicolumn{7}{|l|}{ Control over work } \\
\hline Type of sex & 59.9 & $1.3(0.8$ to 2.1$)$ & $1.1(0.7$ to 1.8$)$ & 75.3 & $1.1(0.7$ to 1.8$)$ & $1.1(0.7$ to 1.8$)$ \\
\hline Amount of money & 65.7 & $2.1^{* *}(1.3$ to 3.3$)$ & $1.8^{*}(1.1$ to 3.1$)$ & 72.8 & $1.1(0.7$ to 1.8$)$ & $1.4(0.9$ to 2.4$)$ \\
\hline
\end{tabular}

${ }^{*} \mathrm{p}<0.05,{ }^{* *} \mathrm{p}<0.01,{ }^{* * *} \mathrm{p}<0.001$.

Adjusted OR control for sample characteristics (age, lack of children, married, geographic proximity, literacy and venue).

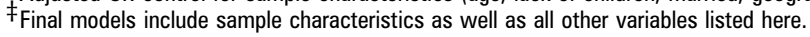

addressing contextual factors that promote this fear so that, over time, FSWs will be more willing to be publicly exposed. Further, in contexts where sex work takes multiple forms, it is important for CMIs, which seek to collectively organise and represent the interests of all groups of FSWs, to be vigilant about identifying groups of FSWs, in this case street-based sex workers, who are under-represented in their activities and developing strategies for including them.

Our study and, therefore, our findings are limited in a number of ways. We have recruited our samples using respondent-driven sampling-a methodology that is meant to produce a random sample from a hidden population. We thus assume, but cannot be certain, that our sample for each round of the survey is representative of FSWs in Rajahmundry; respondent-driven sampling has been identified to be an effective method to sample this population to date. ${ }^{18-20}$

As in most regression analyses, other confounding variables may have affected the associations; however, we controlled for all sample characteristics significantly associated with exposure variables. Also, our findings are based on serial cross-sectional data. As a consequence, we cannot know for certain if the changing composition of the FSW population that is aware of and actively participating in the intervention indicates that the intervention is reaching and bringing in different groups of FSWs, or if the composition of the FSW population itself has changed. Further, if the FSW population has changed, we cannot know for certain if this is a consequence of the intervention activities or unrelated factors. However, we have tried to provide a conceptual framework that suggests plausible reasons to

\section{Key messages}

- Community mobilisation interventions (CMIs) for HIV prevention among female sex workers (FSWs) involve FSWs in publicly visible activities.

- The most important factor associated with both awareness and active utilisation of this CMI was willingness to be identified in public as a FSW.

- Awareness and active utilisation of the CMI increased over time.

- Street-based FSWs were under represented and brothel-based FSWs over represented among both programme aware FSWs and active programme utilisers. believe that the findings, at least in part, reflect intervention activities.

In spite of these limitations, as one of the only systematic analyses of the characteristics of FSWs aware of and actively participating in a CMI, we provide another perspective on intervention implementation, with implications for the impact and sustainability of CMIs.

Contributors KB conceptualised the analysis and interpreted results, wrote the introduction, findings and discussion sections, and made all decisions regarding final content. RB conducted the analysis, produced tables, draughted the methods section and provided input on all other sections of the paper. ER advised on the analysis, assisted with the drafting of the methods and results sections, and provided editorial input on all sections of the paper.

Funding Bill and Melinda Gates Foundation.

\section{Competing interests None.}

Ethics approval This study was conducted with the approval of the IRBs at Duke University, Yale University, YRG Care, India.

Provenance and peer review Not commissioned; externally peer reviewed.

\section{REFERENCES}

1. Ramesha BM, Moses S, Washington R, et al. Determinants of HIV prevalence among female sex workers in four south Indian states: analysis of cross-sectional surveys in twenty-three districts. AIDS 2008;22(Suppl 5):S35-44.

2. Shahmanesh $\mathbf{M}$, Pate $V$, Mabey $D$, et al. Effectiveness of interventions for the prevention of HIV and other sexually transmitted infections in female sex workers in resource poor setting: a systematic review. Trop Med Int Health 2008; 13:659-79.

3. Halli SS, Ramesh BM, O'Neil J, et al. The role of collectives in STI and HIV/AIDS prevention among female sex workers in Karnataka, India. AIDS Care 2006;18:739-49

4. Blankenship KM, West BS, Kershaw TS, et al. Power, community mobilization, and condom use practices among female sex workers in Andhra Pradesh, India. AIDS 2008;22(Suppl 5):S109-16.

5. Basu I, Jana S, Rotheram-Borus MJ, et al. HIV prevention among sex workers in India. J Acquir Immune Defic Syndr 2004;36:845-52.

6. Reza-Paula S, Beattieb T, Syedc HUR, et al. Declines in risk behaviour and sexually transmitted infection prevalence following a community-led HIV preventive intervention among female sex workers in Mysore, India. AIDS 2008:22:91-100.

7. Williams JR, Foss AM, Vickerman $P$, et al. What is the achievable effectiveness of the India AIDS Initiative intervention among female sex workers under target coverage? Model projections from southern India. Sex Transm Infect 2006; 82:372-80

8. Steen R, Mogasale V, Wi T, et al. Pursuing scale and quality in STI interventions with sex workers: initial results from Avahan India AIDS Initiative. Sex Transm Infect 2006;82:381-5.

9. Blankenship KM, Friedman SR, Dworkin S, et al. Structural interventions: concepts, challenges and opportunities for research. J Urban Health 2006;83:59-72.

10. Evans C, Jana S, Lambert H. What makes a structural intervention? Reducing vulnerability to HIV in community settings, with particular reference to sex work. Glob Public Health 2009; http://dx.doi.org/10.1080/17441690902942472

11. Dandona R, Dandona L, Kumar GA, et al. ASCI FPP Study Team. Demography and sex work characteristics of female sex workers in India. BMC Int Health Hum Rights 2006;6:5 
12. Biradavolu M, Burris S, George A, et al. Can sex workers regulate police? Learning from an HIV prevention project for sex workers in southern India. Soc Sci Med 2009;68:1541-7.

13. Cornish F, Ghosh R. The necessary contradictions of 'community-led' health promotion: a case study of HIV prevention in an Indian red light district. Soc Sci Med 2007:64:496-507.

14. Evans C, Lambert H. Implementing community interventions for HIV prevention: insights from project ethnography. Soc Sci Med 2008;66:467-78.

15. Magnani R, Sabin K, Saidel T, et al. Review of sampling hard-to-reach and hidden populations for HIV surveillance. AIDS 2005;19(Suppl 2):S67-72.

16. Semaan S, Lauby J, Liebman J. Street and network sampling in evaluation studies of HIV risk-reduction interventions. AIDS Rev 2002:4:213-23.
17. Heckathorn DD. Respondent driven sampling II: deriving statistically valid population estimates from chain-referral samples of hidden populations. Soc Probl 2002:39:11-34.

18. Yacoubian GS Jr, VanderWall KL, Johnson RJ, et al. Comparing the validity of selfreported recent drug use between adult and juvenile arrestees. J Psychoactive Drugs 2003:35:279-84

19. Semaan S, Santibanez S, Garfein RS, et al. Ethical and regulatory considerations in HIV prevention studies employing respondent-driven sampling. Int J Drug Policy. In Press.

20. Abdul-Quader AS, Heckathorn DD, McKnight C, et al. Effectiveness of respondentdriven sampling for recruiting drug users in New York City: findings from a pilot study. J Urban Health. 2006:83:459-76. 